\title{
PpPIF-1: first isolated full-length $P I F$-like element from the bamboo Phyllostachys pubescens
}

\author{
M.B. Zhou*, X.M. Liu* and D.Q. Tang \\ *These authors contributed equally to this study. \\ Corresponding author: D.Q. Tang \\ E-mail: tang@zafu.edu.cn \\ Genet. Mol. Res. 11 (2): 810-820 (2012) \\ Received March 10, 2011 \\ Accepted July 4, 2011 \\ Published April 3, 2012 \\ DOI http://dx.doi.org/10.4238/2012.April.3.3
}

The Nurturing Station for the State Key Laboratory of Subtropical Silviculture, Zhejiang A \& F University, LinAn, Zhejiang Province, P.R. China

\begin{abstract}
PIF-like elements are the first-described members of a recently discovered and widespread superfamily of DNA transposons, named PIF/Harbinger. Complete and partial PIF-like elements have been isolated from hundreds of plant species. Previously, we identified 139 partial $P I F$-like transposases in the Bambusoideae, of which three were from the bamboo species Phyllostachys pubescens. Here we report identification and isolation of the first full-length $P I F$-like element (PpPIF-1) from P. pubescens; identification was made by chromosome walking, based on a modified magnetic enrichment procedure that allows efficient cloning of flanking sequences up to $3 \mathrm{~kb}$ in length. PpPIF-1 is $5953 \mathrm{bp}$ in length, with 20-bp imperfect inverted terminal repeats and 3-bp target site duplications. This element contains two open reading frames, one encoding a putative transposase, including the complete DDE-domain typical of PIF/Harbinger elements from plants, and the other encoding a DNA-binding protein. There are seven termination codons and two frameshift mutations in the open reading frames, probably due to vertical inactivation.
\end{abstract}

Key words: Phyllostachys pubescens; Full-length PIF-like elements; Chromosome walking 


\section{INTRODUCTION}

Transposable elements are mobile DNA sequences that can move from site to site in the genome, often duplicating during the transposition process and leaving copies at both the donor and recipient loci. Such elements can be assigned to two classes, class I elements (retrotransposons) using an RNA intermediate and class II elements (DNA transposons) moving directly as DNA (Capy et al., 1998; Feschotte et al., 2002a). The mobility of class II elements requires an enzyme named transposase, which is encoded by full-length autonomous elements but can act in trans on partial elements of the same family lacking their own transposase. The enzyme binds in a sequence-specific manner to inverted terminal repeats (ITRs) marking the boundaries of each element, and catalyzes both the DNA cleavage and strand transfer steps of transposition (Feschotte et al., 2002b).

$P$ instability factor $(P I F)$ is an autonomous DNA transposon in maize that was first identified through the analysis of six independent insertions at exactly the same site within intron 2 of the maize $R$ gene (Walker et al., 1997). Elements with similar motifs (PIF-like elements) have recently been allocated to a new and widespread superfamily of DNA transposons called PIF/Harbinger (Zhang et al., 2001, 2004). PIF-like elements share identical ITRs and a strong preference for insertion into the 9-bp palindrome CWCTTAGWG with duplication of the central TTA (Walker et al., 1997; Zhang et al., 2001). The elements contain two open reading frames (ORFs), the first encoding a DNA-binding protein (Jiang et al., 2003; Zhang et al., 2004) and the second encoding a putative transposase featuring the characteristic DDE catalytic motif with the spacing DD(34/37)E. The amino acid sequence surrounding this motif is relatively well conserved in both animals and plants, and has therefore served to establish the evolutionary relationships among PIF-like elements (Zhang et al., 2001, 2004; Casola et al., 2007).

$P I F$-like transposable elements are widespread and diverse in fungi and animals, including nematodes, insects, echinoderms, tunicates, and fish (Kapitonov and Jurka, 1999; Le et al., 2001; Zhang et al., 2001, 2004). In previous study, we have confirmed the existence of many diverse partial PIF-like transposases in the Bambusoideae, particularly in Phyllostachys pubescens (Zhou et al., 2010). Here we report the isolation of the first full-length PIF-like element (PpPIF-1) from P. pubescens by chromosome walking using a modified magnetic enrichment procedure. The putative transposase encoded by this element contains a complete transposase typical of plant $P I F /$ Harbinger elements, as well as a DNA-binding protein.

\section{MATERIAL AND METHODS}

\section{Plant materials and DNA extraction}

Young P. pubescens leaves were collected from the Anji Bamboo Germplasm Garden, Anji, Zhejiang Province of China, and stored in silica gel packs to eliminate moisture. Genomic DNA was extracted using a modified hexadecyltrimethyl-ammonium bromide (CTAB) method (Doyle and Doyle, 1987).

\section{Chromosome walking based on modified magnetic enrichment}

Approximately $2.5 \mu \mathrm{g}$ genomic DNA $>50 \mathrm{~kb}$ in length with minimal smearing was di- 
gested individually with $\mathrm{Dra \textrm {I }}, E c o \mathrm{RV}, P v u \mathrm{II}$, or $S s p \mathrm{I}$ overnight at $37^{\circ} \mathrm{C}$. We removed $5 \mu \mathrm{L}$ from each reaction and separated the products on $0.5 \%$ agarose gel electrophoresis, and then purified the smeared DNA by phenol/chloroform extraction and ethanol precipitation. The resulting blunt DNA fragments were ligated to an adaptor (sense strand 5'-GTA ATA CGA CTC ACT ATA GGG CAC GCG TGG TCG ACG GCC CGG GCT GGT-3', antisense strand 5'-PO - -ACC AGC CC- $\left.\mathrm{N}_{2} \mathrm{H}-3^{\prime}\right)$ at $16^{\circ} \mathrm{C}$ overnight with T4 DNA Ligase (BD Biosciences Clontech, Japan).

Further primers were designed based on the known PIF-like element fragment PhP-3-01 (DQ861540.1) (Zhou et al., 2010). Two sense primers (PhP-GSP-1, 5'-GAC ATG TTC ATG TTG TAG GA-3' and PhP-GSP-2, 5'-CTA CCT CCA TAC CAT TGT GTC C-3') were used to elongate PhP-3-01 from the 3'-end and two antisense primers (PhP-GSP-3, 5'AGC CAG CAC ATA GAC GAA C-3' and PhP-GSP-4, 5'-GTC AAA GTC AAC GGC TGC GAG G-3') were used to elongate PhP-3-01 from the 5'-end. PhP-GSP-1 and PhP-GSP-3 were both biotinylated at the 5'-end. One primer (AP1, 5'-ACT ATA GGG CAC GCG TGG T-3') was designed based on the adaptor sequence and respectively used to be combined with PhP-GSP-2 and PhP-GSP-4 to perform PCR.

The first-round PCR was performed using $50 \mathrm{ng}$ template DNA from each of the four genome walking libraries in a $20-\mu \mathrm{L}$ reaction comprising $0.2 \mathrm{mM}$ PhP-GSP-1 or PhP-GSP-3, $2 \mathrm{U} L A$-Taq polymerase, $1 \mathrm{X}$ PCR buffer (10 mM Tris-HCl, $\mathrm{pH} 8.3,50 \mathrm{mM} \mathrm{KCl}), 1.2 \mathrm{mM}$ $\mathrm{MgCl}_{2}$ and $0.2 \mathrm{mM}$ of each dNTP (TaKaRa, Japan). The reaction was preheated to $94^{\circ} \mathrm{C}$ for 5 min followed by 35 cycles at $94^{\circ} \mathrm{C}$ for $1 \mathrm{~min}, 60^{\circ} \mathrm{C}$ (PhP-GSP-1) or $55^{\circ} \mathrm{C}(\mathrm{PhP}-\mathrm{GSP}-3)$ for 40 $\mathrm{s}, 72^{\circ} \mathrm{C}$ for $5 \mathrm{~min}$, and a final extension step at $72^{\circ} \mathrm{C}$ for $10 \mathrm{~min}$.

The amplification products from both primer extensions were purified using the Dynabeads Kilobase BINDER kit (Invitrogen, USA) according to manufacturer instructions. Briefly, $5 \mu \mathrm{L}$ of the bead suspension was washed twice in the kit's binding buffer and resuspended in $20 \mu \mathrm{L}$ of the same buffer. We then added $20 \mu \mathrm{L}$ of the extension product and incubated the mixture for $3 \mathrm{~h}$ at $25^{\circ} \mathrm{C}$ with gentle agitation. Template genomic DNA was removed by washing in $0.2 \mathrm{M} \mathrm{NaOH}$ (once for $30 \mathrm{~min}$ and four times for $1 \mathrm{~min}$ ), TE buffer (five times for 1 $\mathrm{min}$ ) and double-distilled water (five times for $1 \mathrm{~min}$ ). The purified beads were resuspended in $20 \mu \mathrm{L}$ double-distilled water.

The second-round PCR was performed using $1 \mu \mathrm{L}$ purified template in a $20-\mu \mathrm{L}$ reaction comprising the same components as above but replacing the primers with $0.2 \mathrm{mM} \mathrm{AP} 1$ and $0.2 \mathrm{mM}$ PhP-GSP-2 or PhP-GSP-4. The PCR conditions were the same as above with the exception of different annealing temperatures $\left(60^{\circ} \mathrm{C}\right.$ for $\mathrm{PhP}-\mathrm{GSP}-2$ and $55^{\circ} \mathrm{C}$ for $\mathrm{PhP}-$ GSP-4). Amplification products were resolved by $1 \%$ agarose gel electrophoresis and fragments $>1 \mathrm{~kb}$ in length were purified using the EZ-10 Spin Column DNA Gel Extraction Kit (Biobasic Inc.). The fragments were inserted into vector pCR-XL-TOP (Invitrogen) for cycle sequencing using the BigDye terminator V3.1 system (PE Applied Biosystems) on an ABI PRISM 3100-Avant according to manufacturer instructions.

\section{Southern blot}

Total P. pubescens genomic DNA was digested with HindIII and EcoRI (New England Biolabs Inc.) and fractionated by $0.8 \%$ agarose gel electrophoresis. Positive products amplified with the primers PhP-GSP-2 and AP1 were labeled as probes using the ECL direct nucleic acid labeling system according to manufacturer instructions (GE, USA). After transfer to a nylon mem- 
brane (Hybond- $\mathrm{N}^{+}$, Amersham) and hybridization overnight at $42^{\circ} \mathrm{C}$ with $100 \mathrm{ng}$ probes, membranes were washed twice at $42^{\circ} \mathrm{C}$ for $30 \mathrm{~min}$ in $0.1 \mathrm{X} \mathrm{SSC}, 0.4 \% \mathrm{SDS}$ and $6 \mathrm{M}$ urea, and then for $1 \mathrm{~h}$ in $2 \mathrm{X}$ SSC. Specific bands were detected using the ECL Detection System according to manufacturer instructions and were exposed overnight to X-ray films with an intensifying screen.

\section{Structural and phylogenetic analysis of the full-length PIF-like elements}

The complete $P p P I F-1$ sequence was spliced together at overlap regions in the amplification fragments using Vector NTI 10. Database searches for PIF-like elements were performed using NCBI BLAST (http://www.ncbi.nlm.nih.gov; databases nr, gss, est, and wgs) as well as the TIGR rice genomic database (http://tigrblast.tigr.org/euk-blast/). ITRs were identified using RepeatMasker (http://www.repeatmasker.org/cgi-bin/WEBRepeatMasker). The coding sequences of $P I F$-like transposases were predicted using NetGene2 (http://www. cbs.dtu.dk/services/NetGene2/) and GenScan (http://genes.mit.edu/GENSCAN.html), and the DNA-binding motif was identified by Pfam 24.0 (http://pfam.sanger.ac.uk/).

The PpPIF-1 amino acid sequences were aligned with other PIF sequences using CLUSTALW (Thompson et al., 1994) with default parameters. A phylogenetic tree was generated using the neighbor-joining (NJ), maximum parsimony (MP) and maximum likelihood (ML) methods with the PAUP software v4.0b10 (Swofford, 2002).

\section{RESULTS}

\section{The amplification products were spliced to yield a full-length $P I F$-like element}

We obtained a long sequence by splicing together PhP-3-01 and the two flanking products PhPIF3F and PhPIF5F, which were 3081 and 2644 bp in length, respectively. PhPIF3F was generated using primers AP1 and PhP-GSP-2 (Figure 1A) whereas PhPIF5F was generated using primers AP1 and PhP-GSP-4 (Figure 1B), in both cases with EcoRV-digested genomic DNA as the template. The long sequence was named PpPIF-1, and was $5954 \mathrm{bp}$ in length.
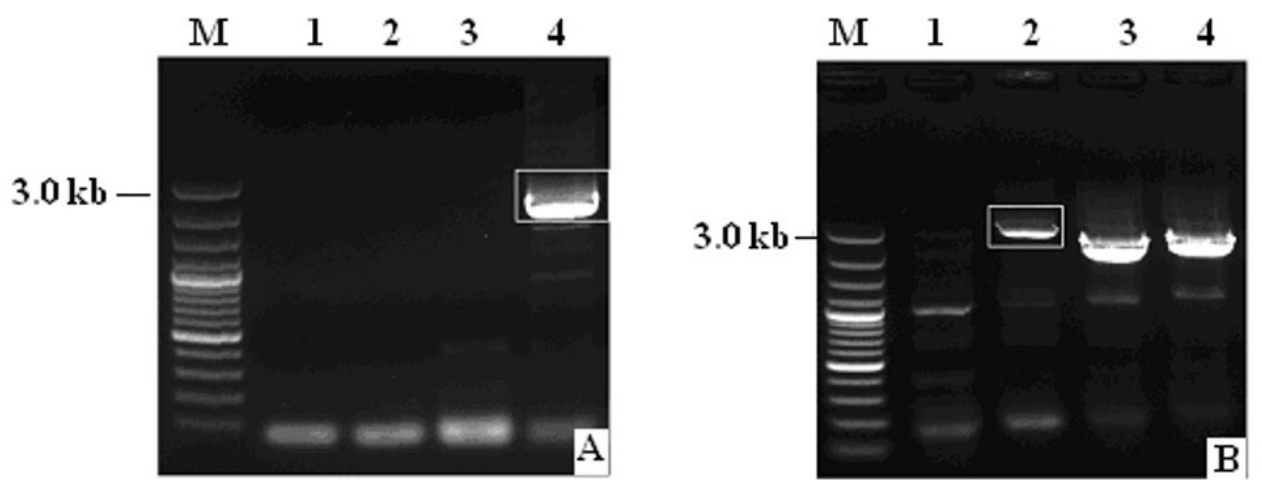

Figure 1. Gel electrophoresis of amplification fragments representing the bamboo PIF-like element $P p P I F-1$. A. The 3'-flanking fragment (PhP3F) amplified with primers AP1 and PhP-GSP-2. B. The 5'-flanking fragment (PhP5F) amplified with primers AP1 and PhP-GSP-4. Lane $M=100$-bp ladder; lanes 1, 2, 3, 4 = genomic DNA templates digested with $D r a \mathrm{I}, E c o$ RV, $P v u \mathrm{II}$, and $S s p \mathrm{I}$, respectively. Positive bands are indicated by rectangular boxes. 
Analysis by BLAST and RepeatMasker revealed that PpPIF-1 contains one fulllength PIF-like element with a 5'-ITR (5'-TAG AGT CTT TGT TTG CCT CA-3') spanning positions -1758 to 1738 and a 3'-ITR (5'-GCA CCC AAC CAA ACG CGC CC-3') spanning positions 3183 to 3203, each contiguous with external TTA/TAA target site duplications. Both ITRs were $20 \mathrm{bp}$ in length but were imperfect repeats with only 12 matching bases (Figures 2 and 3). This structure is very similar to PIF-like elements from Arabidopsis, maize and rice (Table 1) and conforms to the ITR terminal consensus sequence $\mathrm{G}(\mathrm{N})_{5} \mathrm{GTT}$ and target site duplications for known share PIF-like elements (Zhang et al., 2001, 2004).

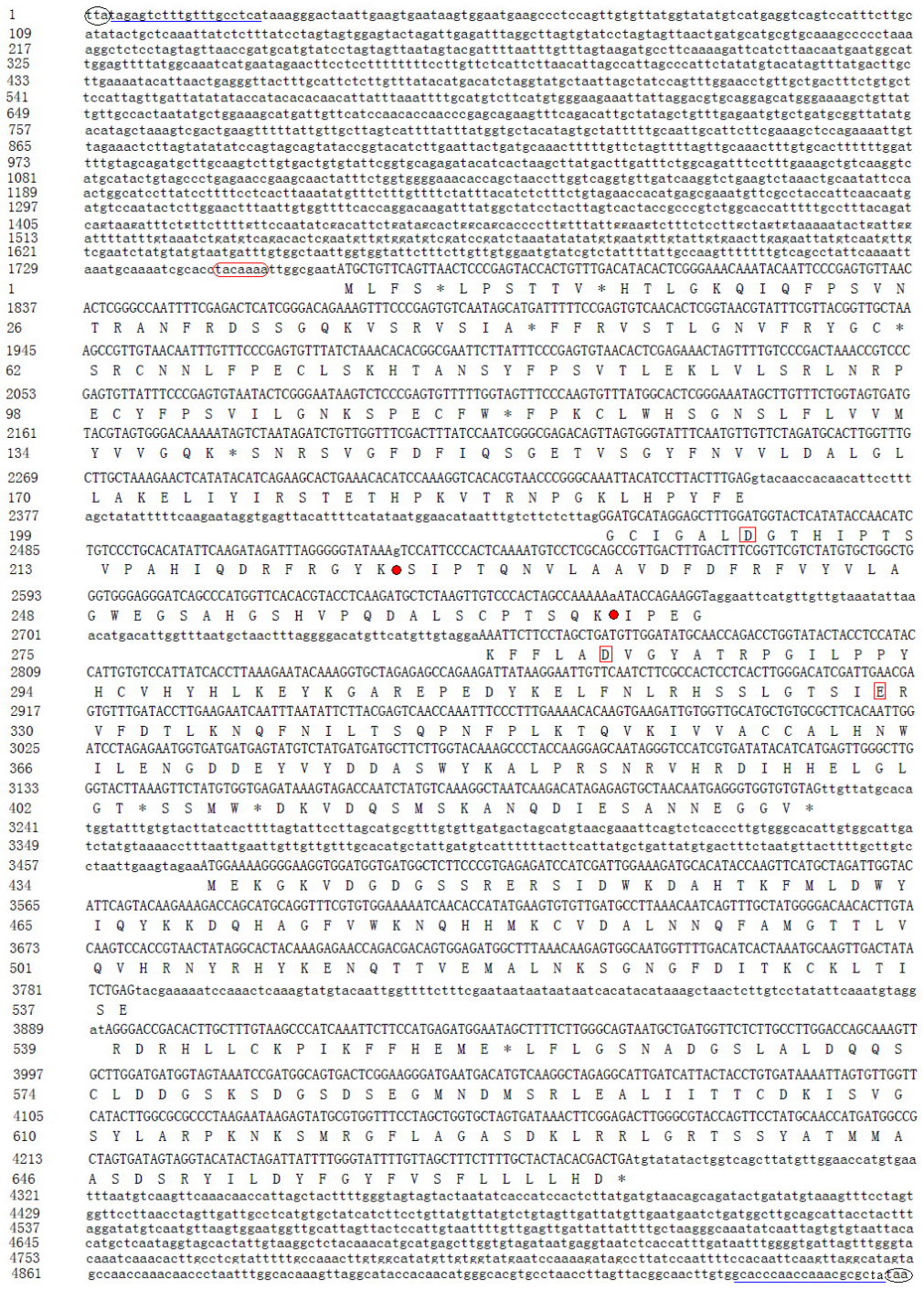

Figure 2. Sequence and structure of $P p P I F-1$. Inverted terminal repeats are underlined in blue with black ovals to indicate the adjacent target site duplications. The acidic residues of the catalytic triad are boxed in red, frameshifts are shown as red ovals and termination codons are identified with asterisks. 


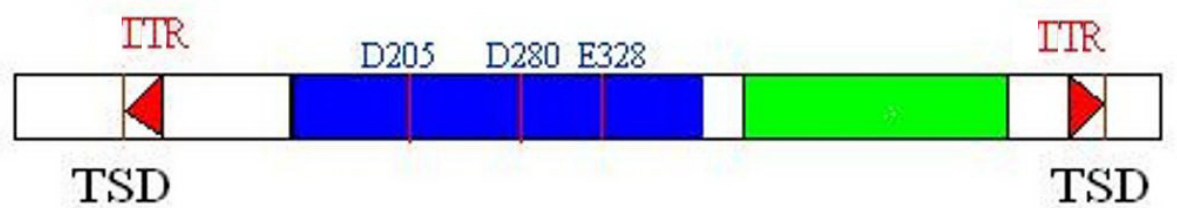

Figure 3. Organization of $P P P I F-1$. The transposase ORF is shown as blue boxes, with the DDE motif identified by red vertical lines. Green box represents ORF1, encoding a DNA-binding protein. Inverted terminal repeats (ITRs) are shown as red triangles and target site duplications (TSDs) are shown as brown vertical lines.

Table 1. Putative target site duplications (TSDs) and inverted terminal repeats (ITRs, length in bp) of PIF-like elements.

\begin{tabular}{lllll}
\hline Species & Name & TSD & ITR (length, sequence) & Reference \\
\hline Z. mays & ZmPIFa & TTA & (14, GGGCCCGTTTGTTT) & Zhang et al., 2001 \\
A. thaliana & AtPIF2 & TTA & (20, GGKGGTGTTATTGGTTAGTG) & Zhang et al., 2001 \\
O. sativa & OsPIF6b & TTA & (21, GGGCCCCTTTGATCGCAGGA) & Zhang et al., 2004 \\
O. sativa & OsPIF25a & TTA & (27, GGGCCTGTTTGGTAGGGCTCCAACTCC) & Zhang et al., 2004 \\
$P$. pubescens & PpPIF-1 & TTA & (20, GGGCGCGTTTGGTTGGGTGC) & \\
\hline
\end{tabular}

In order to investigate the distribution of PPPIF-1 in the bamboo genome, $P$. pubescens genomic DNA were fractioned by the HindIII and EcoRI, which could not cut the probes. Southern blots were probed with the PhPIF3F, revealing several strong bands (5-7 copies) as well as faint smears indicating the presence of at least five copies of this element (Figure 4).

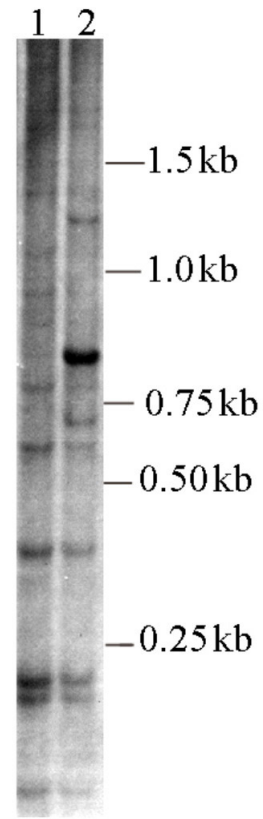

Figure 4. Southern blot of Phyllostachys pubescens genomic DNA probed with PhPIF3F. Lane 1 = DNA digested with HindIII. Lane 2 = DNA digested with EcoRI. Sizes refer to GeneRuler 1-kb molecular ladder. 


\section{$P p P I F-1$ encodes a putative $P I F$-like transposase}

GenScan and Netgene2 analysis showed that PpPIF-1 contains two ORFs (named PhORF1 and PhORF2). PhORF1 spans positions 1-1467, ends in a TAG codon and contains three exons (PhORF1-exon 1-3; 595, 230 and 476 bp, respectively) and two introns (PhORF1intron 1 and 2; 88 and $78 \mathrm{bp}$ in length, respectively). The PhORF1-coding sequence is therefore $1301 \mathrm{bp}$ in length and encodes a 433 -amino acid product. Within the coding sequence there are seven premature termination codons and two frame-shift mutations. PhORF2 is shorter than PhORF1, spanning positions 1710-2522 and ending in a TGA codon. It contains two exons (ORF2-exon 1 and 2; 315 and 393 bp, respectively) and one intron (PhORF2-intron 1; $104 \mathrm{bp}$ ). The PhORF2-coding sequence is therefore $708 \mathrm{bp}$ in length and encodes a product with 236 amino acids. There is a premature termination codon at position 2181 (Figure 2).

We carried out a BLAST search of GenBank to identify sequences matching the two PpPIF-1 ORFs. The closest matches to PhORF1 $\left(\mathrm{E}<1 \times 10^{-38}\right)$ were transposases from the DcMaster (Medicago truncatula), ZmPIFa (maize) and OSPIF1 (rice) elements, each of which belongs to the $P I F /$ Harbinger superfamily. Several representative $P I F$-like transposases were selected from rice and $M$. truncatula and were aligned with $P p P I F-1$ to identify conserved functional domains. PpPIF-1 transposase was most closely related to the transposase from OsTpaseEEC82135, with $58.8 \%$ amino acid sequence identity and preservation of the $\mathrm{H}, \mathrm{N} 2$, $\mathrm{N} 3$, and $\mathrm{C} 1$ blocks, which are conserved in plant PIF-like transposases (Figure 5).

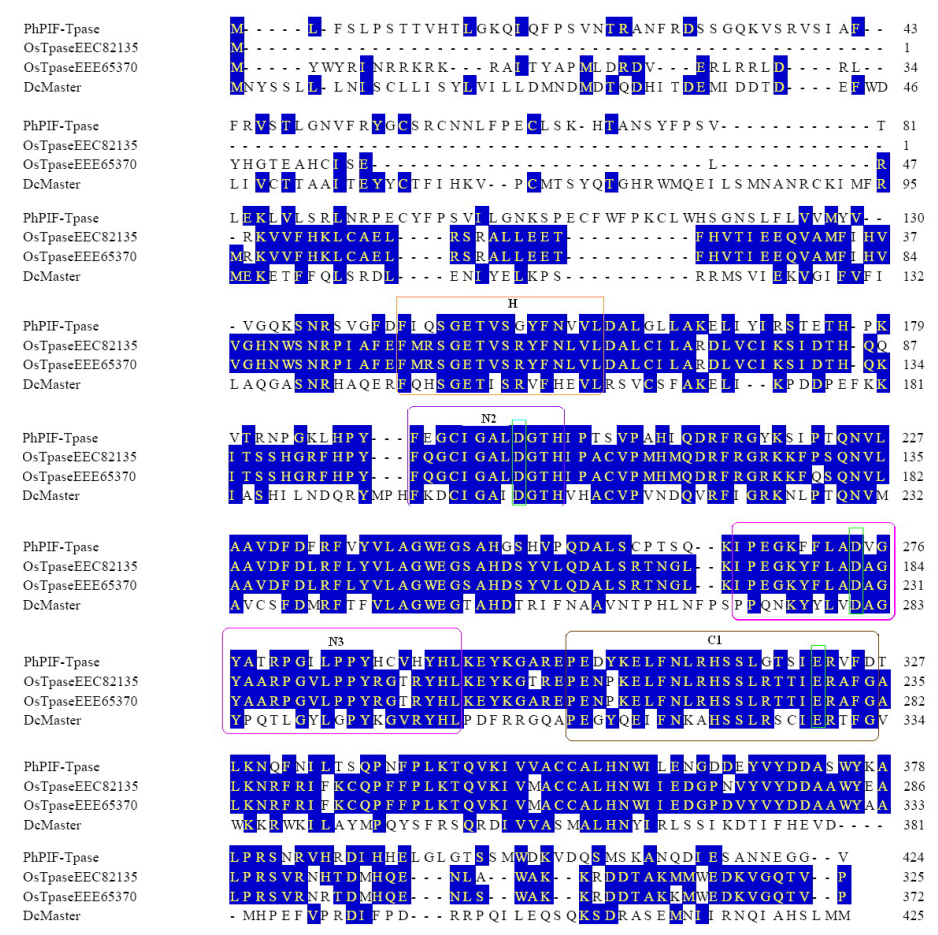

Figure 5. Structural features and conserved amino acid domains of PhORF1 of PhPIF-1. Amino acids that match the consensus are shown with blue shading. Different color rectangles indicate the H, N2, N3, and C1 blocks, respectively. The green rectangle indicates the DD37E motif. 
Pfam 24.0 analysis indicated that PhORF2 is likely to encode a DNA-binding protein $\left(\mathrm{E}<1 \times 10^{-23}\right)$. Translated PhORF2 produced few matches, showing weak homology with DNA-binding protein of other $P I F$-like elements and preserving blocks A and B from plant PIF-like transposases as described by Zhang et al. (2004) (Figure 6).

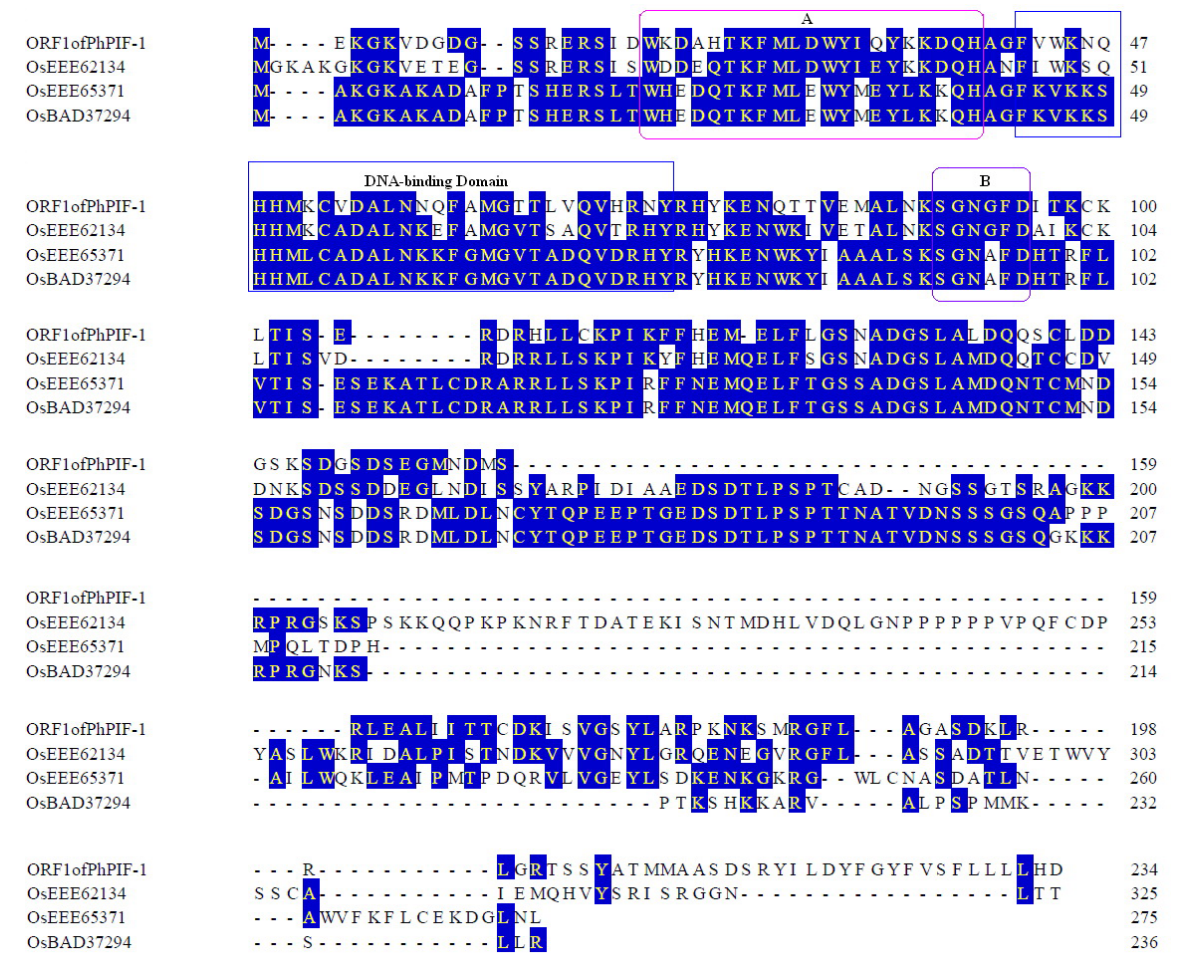

Figure 6. Structural features and conserved amino acid domains of PhORF2 of PpPIF-1. Amino acids that match the consensus are shown with blue shading. Different color rectangles indicate the conserved A and B blocks, respectively. The gray-green rectangle indicates the DNA-binding domain.

\section{PpPIF-1 belongs to subclade A1 of the plant PIF-like elements}

At least four subfamilies of $P I F$-like elements (clades A-D) have been distinguished in plants. Clade A is the largest and most complex, and is further divided into subclades A1-A5 (Zhang et al., 2004). In order to place PpPIF-1 within this phylogenetic tree, it was aligned with sequences representing each of the four subfamilies. Four clusters were defined as the largest and best-supported monophyletic groups, similar to the clades listed above, irrespective of whether the NJ, MP or ML methods was used (bootstrap values $>60 \%$; Figure 7). PpPIF-1 clustered in subclade A1 of the phylogenetic tree with a bootstrap value of $69 \%$, and this placing was also supported by the positioning and intron-exon structure of the ORFs: the putative transposase in $P P P I F-1$ is located upstream from putative DNA-binding protein and contains two introns in conserved positions, features conserved with other subclade A1 PIF/Harbingerlike elements (Zhang et al., 2004). 


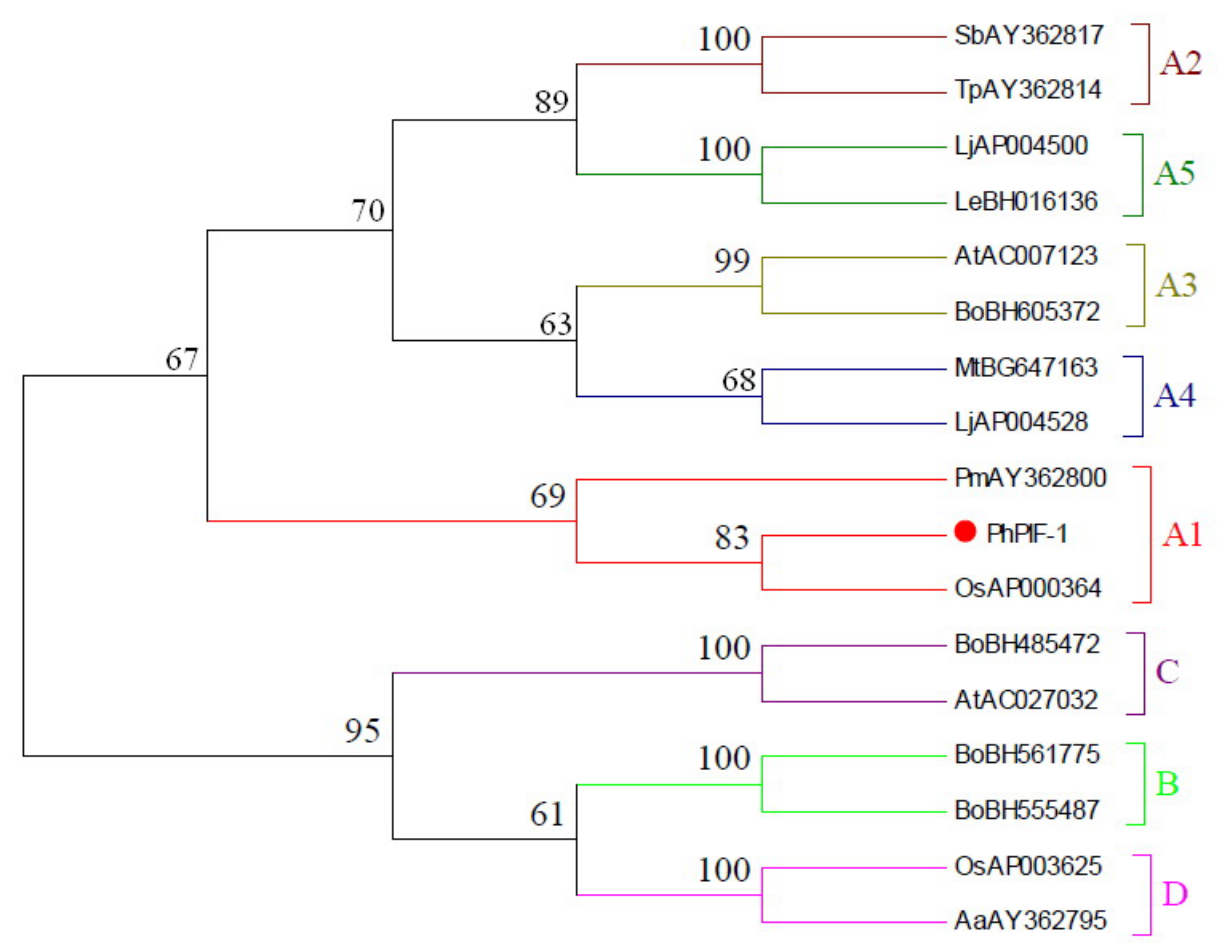

Figure 7. Phylogenetic relationships between $P p P I F-1$ and other representative $P I F$-like sequences collected from GenBank. The phylogenetic tree was generated by aligning 16 catalytic DD motifs from $P I F$-like transposases isolated by PCR (including PhPIF-1) and 15 identified in database searches. Sequences were named according to the convention introduced by Zhang et al. (2004). Main bootstrap values (1000 replicates) are shown.

\section{DISCUSSION}

Previous reports have shown that $P I F$-like elements are widespread and diverse in fungi and animals, including nematodes, insects, echinoderms, tunicates, and fish (Kapitonov and Jurka, 1999; Le et al., 2001; Zhang et al., 2001, 2004). More than 100 PIF-like transposase gene fragments have also been isolated from flowering plants (Zhang et al., 2004), and PIFlike elements are abundant and diverse in the Bambusoideae, particularly the widely cultivated species $P$. pubescens (Zhou et al., 2010). However, only a few full-length PIF-like elements have been identified, such as those found in Arabidopsis (Le et al., 2000), rice (Zhang et al., 2004) and Lotus japonicus (Holligan et al., 2006), due to the availability of genome sequences for these species.

Full-length PIF-like elements in plants may be tens of kilobases in length, which makes them difficult to isolate using normal genome walking procedures. We therefore developed a novel method based on magnetic enrichment to facilitate the amplification of long DNA fragments. In the standard genome walking approach, adaptors are ligated to both ends of each genomic fragment and amplification with adaptor-specific primers generates a background of many non-specific products, which is a significant shortcoming (Sambrook et al., 
1987; Roux, 1995; Siebert et al., 1995). We carried out two rounds of PCR, the first involving only one specific biotinylated primer allowing single biotinylated strands to be enriched by magnetic binding. The second PCR round was then performed using the first-round products as the templates, and with one specific primer and one that annealed to the adaptor. Longdistance polymerases allowed the synthesis of long amplification fragments in both rounds of the procedure, and this facilitated the isolation of targets up to $3 \mathrm{~kb}$ in length, a highly efficient choice for cloning long flanking DNA sequences.

The above method allowed us to isolate the full-length PIF-like element PpPIF-1 from $P$. pubescens. This is the first transposon of the $P I F /$ Harbinger superfamily found in any monocot species other than rice, and the first to be identified experimentally using our modified genome walking method rather than by exploring available sequence data. $P p P I F-1$ is $5953 \mathrm{bp}$ in length and shares many properties with other $P I F$-like elements including short ITRs with the canonical sequence, characteristic 3-bp target site duplications and a putative transposase with qualities similar to other $P I F /$ Harbinger transposases. Phylogenetic analysis indicated that PpPIF-1 belongs to subclade A1 of the PIF/Harbinger superfamily. We believe that the element is inactive due to the accumulation of multiple point mutations in both ORFs (seven termination codons and two frameshift mutations in the transposase ORF and two termination codons in PhORF2), a common evolutionary process known as 'vertical inactivation' (Hartl et al., 1997a,b). Transposable elements may be a more robust target for natural selection than other sequences (Pritham, 2009) because active, autonomous elements reduce the fitness of the host genome through the direct costs of transpositional activity (Brookfield, 1991), causing insertional mutations (Finnegan, 1992) and the promotion of chromosome rearrangements (Montgomery et al., 1987). However, inactive non-autonomous elements may be selectively favored because they facilitate adaptation by providing a basis for unequal exchange during meiosis and therefore increasing genome plasticity (Hartl et al., 1997a,b). Our data provide insight into the structure and diversity of $P I F /$ Harbinger transposons in plants, particularly in bamboo species with few genomic information (Tang, 2009), and add to our knowledge the evolutions of these elements in the bamboo genome.

\section{ACKNOWLEDGMENTS}

Research supported by a special grant from the National Natural Science Foundation of China (\#31170623 and \#31070590) and the "973" Program (grant \#2012CB723008).

\section{REFERENCES}

Brookfield JF (1991). Models of repression of transposition in P-M hybrid dysgenesis by P cytotype and by zygotically encoded repressor proteins. Genetics 128: 471-486.

Capy P, Bazin C, Higuet D and Langin T (1998). Dynamics and Evolution of Transposable Elements. Springer-Verlag, Austin.

Casola C, Lawing AM, Betran E and Feschotte C (2007). PIF-like transposons are common in Drosophila and have been repeatedly domesticated to generate new host genes. Mol. Biol. Evol. 24: 1872-1888.

Doyle JJ and Doyle JL (1987). A rapid isolation procedure for small quantities of fresh leaf materials. Phytochem. Bull. 19: 11-15.

Feschotte C, Jiang N and Wessler SR (2002a). Plant TEs: where genetics meets genomics. Nat. Rev. Genet. 3: 329-341.

Feschotte C, Zhang X and Wessler SR (2002b). Miniature Inverted-Repeat Transposable Elements (MITEs) and their Relationship with Established DNA Transposons. In: Mobile DNA II (Craig NL, Craigie R, Gellert M and Lambowitz 
AM, eds.). Society for Microbiology Press, Washington, 1147-1158.

Finnegan DJ (1992). Transposable Elements. In: The Genome of Drosophila melanogaster (Lindsley DL and Zimm G, eds.). Academic Press, New York, 1096-1107.

Hartl DL, Lohe AR and Lozovskaya ER (1997a). Modern thoughts on an ancyent marinere: function, evolution, regulation. Annu. Rev. Genet. 31: 337-358.

Hartl DL, Lozovskaya ER, Nurminsky DI and Lohe AR (1997b). What restricts the activity of mariner-like TEs? Trends Genet. 13: 197-201.

Holligan D, Zhang XY, Jiang N and Pritham EJ (2006). The transposable element landscape of the model legume Lotus japonicus. Genetics 174: 2215-2228.

Jiang N, Bao Z, Zhang X, Hirochika H, et al. (2003). An active DNA transposon family in rice. Nature 421: 163-167.

Kapitonov VV and Jurka J (1999). Molecular paleontology of transposable elements from Arabidopsis thaliana. Genetica 107: 27-37.

Le QH, Wright S, Yu ZH and Bureau T (2000). Transposon diversity in Arabidopsis thaliana. Proc. Natl. Acad. Sci. U. S. A. 97: 7376-7381.

Le QH, Turcotte K and Bureau T (2001). Tc8, a Tourist-like transposon in Caenorhabditis elegans. Genetics 158: 10811088.

Montgomery EA, Charlesworth B and Langley CH (1987). A test for the role of natural selection in the stabilization of transposable element copy number in a population of Drosophila melanogaster. Genet. Res. 49: 31-41.

Pritham EJ (2009). Transposable elements and factors influencing their success in eukaryotes. Heredity J. 100: 648-655.

Roux KH (1995). Optimization and troubleshooting in PCR. PCR Methods Appl. 4: S185-S194.

Sambrook J, Fritsch EF and Maniatis T (1987). Molecular Cloning: A Laboratory Manual. 2nd edn. Cold Spring Harbor Laboratory, Cold Spring Harbor.

Siebert PD, Chenchik A, Kellogg DE, Lukyanov KA, et al. (1995). An improved PCR method for walking in uncloned genomic DNA. Nucleic Acids Res. 23: 1087-1088.

Swofford DL (2002). PAUP: Phylogenetic Analysis Using Parsimony (*and other Methods). Version 4.0b 10. Sinauer Associates, Sunderland.

Tang DQ (2009). Genomic sequencing and its application for biological and evolutional research in bamboo. Bamboo J. 26: $1-10$.

Thompson JD, Higgins DG and Gibson TJ (1994). CLUSTAL W: improving the sensitivity of progressive multiple sequence alignment through sequence weighting, position-specific gap penalties and weight matrix choice. Nucleic Acids Res. 22: 4673-4680.

Walker EL, Eggleston WB, Demopulos D, Kermicle J, et al. (1997). Insertions of a novel class of transposable elements with a strong target site preference at their locus of maize. Genetics 146: 681-693.

Zhang X, Feschotte C, Zhang Q, Jiang N, et al. (2001). P instability factor: an active maize transposon system associated with the amplification of Tourist-like MITEs and a new superfamily of transposases. Proc. Natl. Acad. Sci. U. S. A. 98: 12572-12577.

Zhang X, Jiang N, Feschotte C and Wessler SR (2004). PIF- and Pong-like transposable elements: distribution, evolution and relationship with Tourist-like miniature inverted-repeat transposable elements. Genetics 166: 971-986.

Zhou MB, Lu JJ, Zhong H and Tang DQ (2010). Distribution and diversity of PIF-like transposable elements in the Bambusoideae subfamily. Plant Sci. 179: 257-266. 